Review Article

\title{
Review on Buccal Drug Delivery System
}

\author{
Shramika A. Chore*, Dr. Sachin. J. Dighade \\ Institute of Pharmacy and Research, Badnera-Amravati, Maharashtra, India. \\ *Corresponding author's E-mail: shramikachore16@gmail.com
}

Received: 19-09-2020; Revised: 25-11-2020; Accepted: 04-12-2020; Published on: 15-12-2020.

\begin{abstract}
Out of various mucosal drug delivery route, the nasal cavity as a site for systemic drug delivery has been investigated by many research groups and the route has already reached commercial status with several drugs including LHRH and calcitonin. However, the potential irritation and the irreversible damage to the ciliary action of the nasal cavity from chronic application of nasal dosage forms, as well as the large intra- and inter-subject variability in mucus secretion in the nasal mucosa, could significantly affect drug absorption from this site. Even though the rectal, vaginal, and ocular mucosa all offer certain advantages, the poor patient acceptability associated with these sites renders them reserved for local applications rather than systemic drug administration. The buccal cavity, on the other hand, is highly acceptable by patients, the mucosa is relatively permeable with a rich blood supply, it is robust and shows short recovery times after stress or damage, and the virtual lack of Langerhans cells makes the buccal mucosa tolerant to potential allergens. Furthermore, buccal transmucosal drug delivery bypasses first pass effect and avoids pre-systemic elimination in the GI tract. These factors make the buccal mucosal cavity a very attractive and feasible site for systemic drug delivery.
\end{abstract}

Keywords: Buccal, buccal mucosal, transmucosal, bioadhesive.

QUICK RESPONSE CODE $\rightarrow$

DOI:

10.47583/ijpsrr.2020.v65i02.022

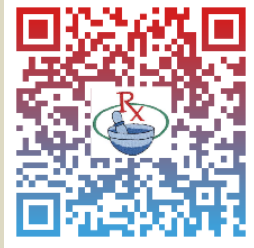

DOI link: http://dx.doi.org/10.47583/ijpsrr.2020.v65i02.022

\section{INTRODUCTION}

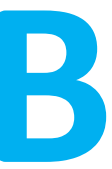

\section{UCCAL DRUG DELIVERY SYSTEM}

The pharmaceutical industry has engendered considerable interest making it a major participant in the healthcare industry. The advances and progress made by pharmaceutical industry have greatly contributed in terms of treatment of disease, thereby enhancing the quality of life. ${ }^{1-3}$ Over the time, scientists and researchers in the drug development industries are focusing on alternate routes of administration to add to the potential of approved drug products, or to overcome the drawbacks of the oral route. Although oral route is preferred for administration of drugs, it is associated with some restrictions for example: hepatic first pass metabolism, local GI toxicity and enzymatic degradation within the GI tract. ${ }^{4}$ One strategy that has been reasonably successful to circumvent such problems is to deliver drugs systemically via an alternate route of administration such as intranasal (IN), buccal/sublingual, pulmonary, or transdermal (TD) With the advances and progress in biotechnology, hydrophilic high molecular weight therapeutic agents such as proteins and peptides are readily available for therapeutic use. However, when administered by the oral route, these agents suffer from problems such as degradation and poor absorption. To overcome these obstacles and for successful delivery of proteins and peptides, the buccal route of drug delivery has acquired significant attention ${ }^{5}$. In view of the systemic transmucosal drug delivery, the buccal mucosa is the preferred region as compared to the sublingual mucosa. One of the reasons is that buccal mucosa is less permeable and is thus not able to elicit a rapid onset of absorption and hence better suited for formulations that are intended for sustained release action. Further, the buccal mucosa being relatively immobile mucosa and readily accessible, it makes it more advantageous for retentive systems used for oral transmucosal drug delivery. To accomplish site-specific drug delivery, a lot of interest has been turned on to the concept of mucoadhesion, which encompasses a pharmaceutical formulation incorporating mucoadhesive hydrophilic polymers along with the active pharmaceutical ingredient (API). The rationale being that the formulation will be 'held' on a biological surface for localized drug delivery and the release of API will be close to the site of action leading to enhanced bioavailability.

\section{Advantages of Buccal Drug Delivery System: ${ }^{11}$}

Drug administration via buccal mucosa offers several distinct advantages:

1. The buccal mucosa is relatively permeable with a rich blood supply, robust in comparison to the other mucosal tissues.

2. Bypass the first-pass effect and non-exposure of the drugs to the gastrointestinal fluids. 
3. Easy access to the membrane sites so that the delivery system can be applied, localized and removed easily.

4. Improve the performance of many drugs, as they are having prolonged contact time with the mucosa.

5. High patient acceptance compared to other non-oral routes of drug administration.

6. Tolerance (in comparison with the nasal mucosa and skin) to potential sensitizers.

7. Increased residence time combined with controlled API release may lead to lower administration frequency.

\section{Disadvantages of Buccal Drug Delivery System: ${ }^{11}$}

The main disadvantages of buccal administration are:

1. Limited absorption area- the total surface area of the membranes of the oral cavity available for drug absorption is $170 \mathrm{~cm}^{2}$ of which50 $\mathrm{cm}^{2}$ represents non keratinized tissues, including buccal membrane.

\section{Barrier properties of the mucosa.}

3. The continuous secretion of the saliva (0.5-2 l/day) leads to subsequent dilution of the drug.

4. The hazard of choking by involuntarily swallowing delivery system is a concern.

5. Saliva Swallowing can also potentially lead to the loss of dissolved or suspended drug and ultimately the involuntary removal of the dosage form.

\section{Anatomy and Physiology: $:^{12,13}$}

The buccal mucosa is composed of an outermost layer of stratified squamous epithelium (Fig-1). Below this lies a basement membrane, a lamina propria followed by the submucosa as the innermost layer. The epithelium is similar to stratified squamous epithelia found in the rest of the body in that it has a mitotically active basal cell layer, advancing through a number of differentiating intermediate layers to the superficial layers, where cells are shed from the surface of the epithelium. The epithelium of the buccal mucosa is about 40-50 cell layers thick, while that of the sublingual epithelium contains somewhat fewer. The epithelial cells increase in size and become flatter as they travel from the basal layers to the superficial layers.

The turnover time for the buccal epithelium has been estimated at 5-6 days, and this is probably representative of the buccal mucosa as a whole. The buccal mucosal thickness varies depending on the site: the buccal mucosa measures at $500-800 \mu \mathrm{m}$, while the mucosal thickness of the hard and soft palates, the floor of the mouth, the ventral tongue, and the gingivae measure at about 100$200 \mu \mathrm{m}$. The composition of the epithelium also varies depending on the site in the buccal cavity. The mucosa of areas subject to mechanical stress (the gingivae and hard palate) is keratinized similar to the epidermis. The mucosa of the soft palate, the sublingual, and the buccal regions, however, are not keratinized. ${ }^{14}$
The keratinized epithelia contain neutral lipids like ceramides and acylceramides which have been associated with the barrier function. These epithelia are relatively impermeable to water. In contrast, non-keratinized epithelia, such as the floor of the mouth and the buccal epithelia, do not contain acylceramides and only have small amounts of ceramide. They also contain small amounts of neutral but polar lipids, mainly cholesterol sulfate and glucosyl ceramides. These epithelia have been found to be considerably more permeable than water than keratinized epithelia. ${ }^{15,16}$

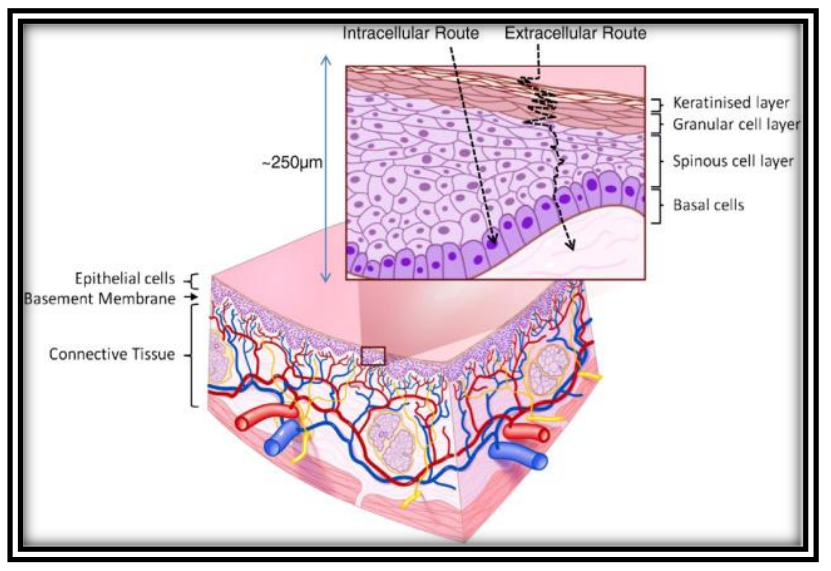

Figure 1: The Structure of the Buccal Mucosa ${ }^{17}$

Fig. 1 also shows different routes by which drugs can cross the buccal mucosa. Drugs which act upon keratinocyte cell surface receptors or those which are intended to have an action in the connective tissue or are for systemic delivery would ideally cross the epithelium via the extracellular route without being internalised by the cells of the epithelium. Drugs and therapeutic agents which act upon intracellular targets within the epithelium should be easily internalized by cells and retained within the epithelium.

\section{Permeability: ${ }^{18-22}$}

The buccal mucosa in general is a somewhat leaky epithelia intermediate between that of the epidermis and intestinal mucosa. It is estimated that the permeability of the buccal mucosa is $4-4000$ times greater than that of the skin. As indicative by the wide range in this reported value, there are considerable differences in permeability between different regions of the buccal cavity because of the diverse structures and functions of the different buccal mucosa. In general, the permeabilities of the buccal mucosa decrease in the order of sublingual greater than buccal and buccal greater than palatal. This rank order is based on the relative thickness and degree of keratinization of these tissues, with the sublingual mucosa being relatively thin and non-keratinized, the buccal thicker and non-keratinized.

It is currently believed that the permeability barrier in the buccal mucosa is a result of intercellular material derived from the so-called 'membrane coating granules' (MCG). When cells go through differentiation, MCGs start forming and at the apical cell surfaces they fuse with the plasma membrane and their contents are discharged into the 
intercellular spaces at the upper one third of the epithelium. This barrier exists in the outermost $200 \mu \mathrm{m}$ of the superficial layer.

Permeation studies have been performed using a number of very large molecular weight tracers, such as horseradish peroxidase and lanthanum nitrate. When applied to the outer surface of the epithelium, these tracers penetrate only through outermost layer or two of cells. When applied to the submucosal surface, they permeate up to, but not into, the outermost cell layers of the epithelium. According to these results, it seems apparent that flattened surface cell layers present the main barrier to permeation, while the more isodiametric cell layers are relatively permeable. In both keratinized and non-keratinized epithelia, the limit of penetration coincided with the level where the MCGs could be seen adjacent to the superficial plasma membranes of the epithelial cells. Since, the same result was obtained in both keratinized and non-keratinized epithelia, keratinization by itself is not expected to play a significant role in the barrier function. The components of the MCGs in keratinized and non-keratinized epithelia are different. The MCGs of keratinized epithelium are composed of lamellar lipid stacks, whereas the nonkeratinized epithelium contains MCGs that are nonlamellar. The MCG lipids of keratinized epithelia include sphingomyelin, glucosylceramides, ceramides, and other nonpolar lipids, however for non-keratinized epithelia, the major MCG lipid components are cholesterol esters, cholesterol, and glycosphingolipids. Aside from the MCGs, the basement membrane may present some resistance to permeation as well, however the outer epithelium is still considered to be the rate limiting step to mucosal penetration. The structure of the basement membrane is not dense enough to exclude even relatively large molecules. ${ }^{23-25}$

\section{Environment: ${ }^{26-28}$}

The cells of the buccal epithelia are surrounded by an intercellular ground substance, mucus, the principle components of which are complexes made up of proteins and carbohydrates. These complexes may be free of association or some maybe attached to certain regions on the cell surfaces. This matrix may actually play a role in cellcell adhesion, as well as acting as a lubricant, allowing cells to move relative to one another. Along the same lines, the mucus is also believed to play a role in bioadhesion of mucoadhesive drug delivery systems. In stratified squamous epithelia found elsewhere in the body, mucus is synthesized by specialized mucus secreting cells like the goblet cells, however in the buccal mucosa, mucus is secreted by the major and minor salivary glands as part of saliva.

Up to $70 \%$ of the total mucin found in saliva is contributed by the minor salivary glands. At physiological $\mathrm{pH}$ the mucus network carries a negative charge (due to the sialic acid and sulfate residues) which may play a role in mucoadhesion. At this $\mathrm{pH}$ mucus can form a strongly cohesive gel structure that will bind to the epithelial cell surface as a gelatinous layer.

Another feature of the environment of the buccal cavity is the presence of saliva produced by the salivary glands. Saliva is the protective fluid for all tissues of the buccal cavity. It protects the soft tissues from abrasion by rough materials and from chemicals. It allows for the continuous mineralization of the tooth enamel after eruption and helps in demineralization of the enamel in the early stages of dental caries. Saliva is an aqueous fluid with $1 \%$ organic and inorganic materials. The major determinant of the salivary composition is the flow rate which in turn depends upon three factors: the time of day, the type of stimulus, and the degree of stimulation. The salivary $\mathrm{pH}$ ranges from 5.5 to 7 depending on the flow rate. At high flow rates, the sodium and bicarbonate concentrations increase leading to an increase in the $\mathrm{pH}$. The daily salivary volume is between 0.5 to 2 liters and it is this amount of fluid that is available to hydrate buccal mucosal dosage forms. A main reason behind the selection of hydrophilic polymeric matrices as vehicles for buccal transmucosal drug delivery systems is this water rich environment of the buccal cavity.

\section{Buccal Routes of Drug Absorption:29,30}

The are two permeation pathways for passive drug transport across the buccal mucosa: paracellular and transcellular routes. Permeants can use these two routes simultaneously, but one route is usually preferred over the other depending on the physicochemical properties of the diffusion. Since the intercellular spaces and cytoplasm are hydrophilic in character, lipophilic compounds would have low solubilities in this environment. The cell membrane, however, is rather lipophilic in nature and hydrophilic solutes will have difficulty permeating through the cell membrane due to a low partition coefficient.

Therefore, the intercellular spaces pose as the major barrier to permeation of lipophilic compounds and the cell membrane acts as the major transport barrier for hydrophilic compounds. Since the buccal epithelium is stratified, solute permeation may involve a combination of these two routes. The route that predominates, however, is generally the one that provides the least amount of hindrance to passage.

\section{Buccal Mucosa As A Site For Drug Delivery: ${ }^{13,14,31,32}$}

As stated above, there are three different categories of drug delivery within the buccal cavity (i.e., sublingual, buccal, and local drug delivery). Selecting one over another is mainly based on anatomical and permeability differences that exist among the various buccal mucosal sites. The sublingual mucosa is relatively permeable, giving rapid absorption and acceptable bioavailability's of many drugs, and is convenient, accessible, and generally well accepted. The sublingual route is by far the most widely studied of these routes. Sublingual dosage forms are of two different designs, those composed of rapidly disintegrating tablets, and those consisting of soft gelatin capsules filled with liquid drug. Such systems create a very 
high drug concentration in the sublingual region before they are systemically absorbed across the mucosa. The buccal mucosa is considerably less permeable than the sublingual area, and is generally not able to provide the rapid absorption and good bioavailability's seen with sublingual administration. Local delivery to tissues of the buccal cavity has a number of applications, including the treatment of toothaches, periodontal disease, bacterial and fungal infections, aphthous and dental stomatitis, and in facilitating tooth movement with prostaglandins.

Even though the sublingual mucosa is relatively more permeable than the buccal mucosa, it is not suitable for an buccal transmucosal delivery system. The sublingual region lacks an expanse of smooth muscle or immobile mucosa and is constantly washed by a considerable amount of saliva making it difficult for device placement. Because of the high permeability and the rich blood supply, the sublingual route is capable of producing a rapid onset of action making it appropriate for drugs with short delivery period requirements with infrequent dosing regimen.

Due to two important differences between the sublingual mucosa and the buccal mucosa, the latter is a more preferred route for systemic transmucosal drug delivery. First difference being in the permeability characteristics of the region, where the buccal mucosa is less permeable and is thus not able to give a rapid onset of absorption (i.e., more suitable for a sustained release formulation). Second being that, the buccal mucosa has an expanse of smooth muscle and relatively immobile mucosa which makes it a more desirable region for retentive systems used for buccal transmucosal drug delivery. Thus the buccal mucosa is more fitted for sustained delivery applications, delivery of less permeable molecules, and perhaps peptide drugs.

Similar to any other mucosal membrane, the buccal mucosa as a site for drug delivery has limitations as well. One of the major disadvantages associated with buccal drug delivery is the low flux which results in low drug bioavailability. Various compounds have been investigated for their use as buccal penetration enhancers in order to increase the flux of drugs through the mucosa. Since, the buccal epithelium is similar in structure to other stratified epithelia of the body, enhancers used to improve drug permeation in other absorptive mucosa have been shown to work in improving buccal drug penetration. Drugs investigated for buccal delivery using various permeation/absorption enhancers range in both molecular weight and physicochemical properties. Small molecules such as butyric acid and butanol, ionizable low molecular weight drugs such as Acyclovir, Propranolol, and salicylic acid, large molecular weight hydrophilic polymers such as dextran's, and a variety of peptides including luteinizing hormone releasing hormone (LHRH), insulin, and $\alpha$-interferon have all been studied.

\section{Bioadhesive polymers: ${ }^{33-36}$}

Other than the low flux associated with buccal mucosal delivery, a major limitation of the buccal route of administration is the lack of dosage form retention at the site of absorption. Consequently, bioadhesive polymers have extensively been employed in buccal drug delivery systems.

Bioadhesive polymers are defined as polymers that can adhere onto a biological substrate. The term mucoadhesion is applied when the substrate is mucosal tissue.

Polymers which can adhere to either hard or soft tissue have been used for many years in surgery and dentistry. Diverse classes of polymers have been investigated for their potential use as mucoadhesives.

These include synthetic polymers such as monomeric polyacrylic acid, hydroxypropyl methylcellulose, and poly methacrylate derivatives as well as naturally occurring polymers such as hyaluronic acid and chitosan. Other synthetic polymers such as carbopol, polycarbophils, epoxy resins, polystyrene, and natural-product cement have also been extensively investigated.

In general, dosage forms designed for buccal administration should not cause irritation and should be small and flexible enough to be accepted by the patient. These requirements can be met by using hydrogels. Hydrogels are hydrophilic matrices that are capable of swelling when placed in aqueous media. Normally, hydrogels are cross linked so that they would not dissolve in the medium and would only absorb water. When drugs are loaded into these hydrogels, as water is absorbed into the matrix, chain relaxation occurs and drug molecules are released through the spaces or channels within the hydrogel network. In a more broad meaning of the term, hydrogels would also include water-soluble matrices that are capable of swelling in aqueous media, these include natural gums and cellulose derivatives. These 'pseudohydrogels' swell infinitely and the component molecules dissolve from the surface of the matrix.

Drug release would then occur through the spaces or channels within the network as well as through the dissolution and/or the disintegration of the matrix. The use of hydrogels as adhesive preparations for transmucosal drug delivery has acquired considerable attention in recent years.

The buccal mucosa offers several advantages for controlled drug delivery for extended periods of time. The mucosa is well supplied with both vascular and lymphatic drainage and first-pass metabolism in the liver and presystemic elimination in the gastrointestinal tract are avoided. The area is well suited for a retentive device and appears to be acceptable to the patient. With the right dosage form design and formulation, the permeability and the local environment of the mucosa can be controlled in order to accommodate drug permeation. Buccal drug 
delivery is a promising area for continued research with the aim of systemic delivery of orally inefficient drugs as well as a feasible and attractive alternative for noninvasive delivery of potent peptide and protein drug molecules. However, the need for safe and effective buccal permeation/absorption enhancers is a crucial component for a prospective future in the area of buccal drug delivery.

\section{FORMULATION ASPECTS \& DOSAGE FORMS FOR BUCCAL} DRUG DELIVERY ${ }^{11,12,37}$

Other than the low flux associated with buccal mucosal delivery, a major limitation of the buccal route of administration is the lack of dosage form retention at the site of absorption. Consequently, bioadhesive polymers have extensively been employed in buccal drug delivery systems. Bioadhesive polymers are defined as polymers that can adhere onto a biological substrate. The term mucoadhesion is applied when the substrate is mucosal tissue. Polymers which can adhere to either hard or soft tissue have been used for many years in surgery and dentistry. Diverse classes of polymers have been investigated for their potential use as mucoadhesives. These include synthetic polymers such as monomeric cyanoacrylate, polyacrylic acid, hydroxypropylmethylcellulose, and poly methacrylate derivatives as well as naturally occurring polymers such as hyaluronic acid and chitosan. Other synthetic polymers such as polyurethanes, epoxy resins, polystyrene, and natural-product cement have also been extensively investigated.

In general, dosage forms designed for buccal administration should not cause irritation and should be small and flexible enough to be accepted by the patient. These requirements can be met by using hydrogels. Hydrogels are hydrophilic matrices that are capable of swelling when placed in aqueous media. Normally, hydrogels are cross linked so that they would not dissolve in the medium and would only absorb water. When drugs are loaded into these hydrogels, as water is absorbed into the matrix, chain relaxation occurs and drug molecules are released through the spaces or channels within the hydrogel network. In a more broad meaning of the term, hydrogels would also include water-soluble matrices that are capable of swelling in aqueous media, these include natural gums and cellulose derivatives. These 'pseudohydrogels' swell infinitely and the component molecules dissolve from the surface of the matrix. Drug release would then occur through the spaces or channels within the network as well as through the dissolution and/or the disintegration of the matrix. The use of hydrogels as adhesive preparations for transmucosal drug delivery has acquired considerable attention in recent years.

Bioadhesive formulations use polymers as the adhesive component. These formulations are often water soluble and when in a dry form attract water from the biological surface and this water transfer leads to a strong interaction. These polymers also form viscous liquids when hydrated with water that increases their retention time over mucosal surfaces and may lead to adhesive interactions. Bioadhesive polymers should possess certain physicochemical features including hydrophilicity, numerous hydrogen bond-forming groups, flexibility for interpenetration with mucus and epithelial tissue, and visco-elastic properties.

\section{Ideal characteristics of polymers for Buccal Drug Delivery Systems:}

$\checkmark$ Polymer and its degradation products should be nontoxic, non-irritant and free from leachable impurities.

$\checkmark$ Should have good spreadability, wetting, swelling and solubility and biodegradability properties.

$\checkmark \mathrm{pH}$ should be biocompatible and should possess good viscoelastic properties.

$\checkmark$ Should adhere quickly to buccal mucosa and should possess sufficient mechanical strength.

$\checkmark$ Should possess peel, tensile and shear strengths at the bioadhesive range.

$\checkmark$ Polymer must be easily available and its cost should not be high.

$\checkmark$ Should show bioadhesive properties in both dry an liquid state.

$\checkmark$ Should demonstrate local enzyme inhibition and penetration enhancement properties.

$\checkmark$ Should demonstrate acceptable shelf life.

$\checkmark$ Should have optimum molecular weight.

$\checkmark$ Should possess adhesively active groups.

$\checkmark$ Should have required spatial conformation.

$\checkmark$ Should be sufficiently cross-linked but not to the degree of suppression of bond forming groups.

$\checkmark$ Should not aid in development of secondary infections such as dental caries.

Over the past few years, different dosage forms intended for buccal drug delivery have been developed. Buccal mucoadhesive dosage forms can be categorized into three types:

Type I- It is a single layer device with multidirectional drug release. This type of

dosage form suffers from significant drug loss due to swallowing.

Type II- In this type, an impermeable backing layer is superimposed on top of the drug loaded bioadhesive layer creating a double-layered device and preventing drug loss from the top surface of the dosage form into the oral cavity.

Type III- This is a unidirectional release device, from which drug loss is minimal, since the drug is released only from 
the side adjacent to the buccal mucosa. This can be achieved by coating every face of the dosage form, except the one that is in contact with the buccal mucosa. Buccal dosage forms can also be classified as either a "reservoir" or "matrix" type.

A number of relevant buccal mucoadhesive dosage forms have been developed for a variety of drugs. Several peptides, including Thyrotropin-Releasing Hormone (TRH), Insulin, Protirelin, Buserelin and Oxytocin, have been delivered via the buccal route, also drugs with relatively low bioavailability can be delivered via buccal route. Buccal dosage forms can be used to treat both local and systemic conditions.

Table 1. summarizes various buccal dosage forms

Table 1: Various buccal dosage forms

\begin{tabular}{|c|c|c|c|c|}
\hline Dosage Forms & Structures & Release & Effect & Active ingredients \\
\hline Matrix tablets & $\begin{array}{l}\text { Monolithic matrix, } \\
\text { Coating matrix (coated on the } \\
\text { outer side or on all but one } \\
\text { faces), } \\
\text { Two-layer matrix, } \\
\text { Two-layer matrix coated with } \\
\text { impermeable layer }\end{array}$ & $\begin{array}{l}\text { Sustained or } \\
\text { bidirectional } \\
\text { Monodirectional } \\
\text { Bidirectional } \\
\text { Monodirectional }\end{array}$ & $\begin{array}{l}\text { Local } \\
\text { (mainly) } \\
\text { Systemic }\end{array}$ & $\begin{array}{l}\text { Local administration: metronidazole. } \\
\text { Systemic administration: propanolol, } \\
\text { timolol, metoclopramide, morphine } \\
\text { sulphate, nitroglycerin, codein, } \\
\text { insulin, calcitonin }\end{array}$ \\
\hline Patches & $\begin{array}{l}\text { Laminated film with coating } \\
\text { Layer }\end{array}$ & Monodirectional & $\begin{array}{l}\text { Local or } \\
\text { systemic }\end{array}$ & $\begin{array}{l}\text { Local administration: } \\
\text { diclofenac, tannic acid, boric acid. } \\
\text { Systemic administration: TRH, } \\
\text { oxytocin, octreotide, }\end{array}$ \\
\hline Lipophilic gels & $\begin{array}{l}\text { Cubic and lamellar liquid } \\
\text { crystalline phases of } \\
\text { Glycerylmonooleate }\end{array}$ & - & Systemic & $\begin{array}{l}\text { Systemic administration: } \\
\text { enkephalin }\end{array}$ \\
\hline Transfersomes & $\begin{array}{l}\text { Phospholipids deformable } \\
\text { Vesicles }\end{array}$ & - & Systemic & $\begin{array}{l}\text { Systemic administration: } \\
\text { insulin }\end{array}$ \\
\hline
\end{tabular}

\section{Buccal Patches:}

Buccal patches are described as laminates which comprise of an impermeable backing layer, a drug containing reservoir layer which releases the drug in a controlled manner, and a bioadhesive surface for mucosal attachment. Two methods, namely, solvent casting method and direct milling are used to prepare adhesive patches. In the solvent casting method, the intermediate sheet from which patches are punched is prepared by casting the solution of the drug and polymer(s) onto a backing layer sheet, and subsequently allowing the solvent(s) to evaporate.

\section{Buccal Films:}

In recent times, a number of mucoadhesive dosage forms for buccal drug delivery have been developed such as tablet, films, patches, discs, ointments and gels. However, buccal films are preferable over mucoadhesive discs and tablets in terms of patient comfort and flexibility and they ensure more accurate drug dosing and longer residence time compared to gels and ointments. Buccal films also reduce pain by protecting the wound surface and hence increase the treatment effectiveness.

\section{Buccal Gels and Ointments:}

These are semisolid dosage forms having the advantage of easy dispersion throughout the oral mucosa. The problem of poor retention of gels at the application site has been overcome by using bioadhesive formulations. Certain bioadhesive polymers for example, sodium carboxymethylcellulose undergo a phase change from a liquid to a semisolid. This change enhances or improves the viscosity, resulting in sustained or controlled release of drugs.

\section{Microparticles:}

Bioadhesive microparticles offer the same advantages as tablets but their physical properties enable them to make intimate contact with a lager mucosal surface area. In addition, they can also be delivered to less accessible sites including the GI tract and upper nasal cavity. The small size of microparticles compared with tablets means that they are less likely to cause local irritation at the site of adhesion and the uncomfortable sensation of a foreign object within the oral cavity is reduced. 


\section{Wafers:}

Bromberg et al. described a conceptually novel periodontal drug delivery system that is intended for the treatment of microbial infections associated with peridontitis. The delivery system is a composite wafer with surface layers possessing adhesive properties, while the bulk layer consists of antimicrobial agents, biodegradable polymers and matrix polymers.

\section{Lozenges:}

Bioadhesive lozenges may be used for the delivery of drugs that act topically within the mouth including antimicrobials, corticosteroids, local anesthetics, antibiotics and antifungals. Conventional lozenges produce a high initial release of drug in the oral cavity, which rapidly declines to sub therapeutic levels, thus multiple daily dosing is required. A slow release bioadhesive lozenge offers the potential for prolonged drug release with improved patient compliance. Cod and Daisy investigated bioadhesive lozenges as a means to deliver antifungal agents to the oral cavity

\section{Buccal Tablets: ${ }^{2,11}$}

Tablets have been the most commonly investigated dosage form for buccal drug delivery. Buccal tablets are small, flat, and oval shaped dosage form and unlike conventional tablets allow for drinking and speaking without major discomfort. They soften, adhere to the mucosa and are retained in position until dissolution and/or release is complete. Monolithic and two-layered matrix tablets have been designed for buccal drug delivery. Bioadhesive tablets may be prepared using different methods such as direct compression or wet granulation technique. For buccal drug delivery, the tablets which are inserted into the buccal pouch may dissolve or erode; therefore, they must be formulated and compressed with sufficient pressure only to give a hard tablet. To enable or to achieve unidirectional release of drug, water impermeable materials, such as ethyl cellulose, hydrogenated castor oil, etc. may be used either by compression or by spray coating to coat every face of the tablet except the one that is in contact with the buccal mucosa. If necessary, the drug may be formulated in certain physical states, such as microspheres, prior to direct compression in order to achieve some desirable properties, e.g. enhanced activity and prolonged drug release. Several bioadhesive tablet formulations were developed in recent years either for local or systemic drug delivery. Tablets that are placed directly onto the mucosal surface have been demonstrated to be excellent bioadhesive formulations. However, size is a limitation for tablets due to the requirement for the dosage form to have intimate contact with the mucosal surface. These tablets adhere to the buccal mucosa in presence of saliva. They are designed to release the drug either unidirectional targeting buccal mucosa or multidirectional in to the saliva.

Buccal drug delivery is the simplest and easiest way of administering drugs. Because of the greater stability, smaller bulk, accurate dosage and easy production, solid buccal dosages forms have many advantages over other types of buccal dosage forms. Therefore, most of the new chemical entities (NCE) under development these days are intended to be used as a solid dosage form that originate an effective and reproducible in-vivo plasma concentration after buccal administration.

Tablets may be defined as solid pharmaceutical dosage forms containing medicament with or without suitable excipients and prepared either by compression or moulding. Despite the long and continuing history of the development of new technologies for administration of drugs, the tablet form remains the most commonly used dosage form.

The best new therapeutic entity in the world is of little value without an appropriate delivery system. Tableted drug delivery systems can range from relatively simple immediate release formulations to complex extended or modified release dosage forms. The most important role of a drug delivery system is to get the drug "delivered" to the site of action in sufficient amount and at the appropriate rate; however, it must also meet a number of other essential criteria.

\section{Properties of an Ideal Buccal Tablet: ${ }^{2}$}

The objective of formulation and fabrication of tablet is to deliver the correct amount of drug in proper form at or over proper time.

$\square$ Tablet should be elegant having its own identity and free from defects such as cracks, chips, contamination, discoloration etc.

$\square$ It should have chemical and physical stability to maintain its physical integrity over time.

It should be capable to prevent any alteration in the chemical and physical properties of medicinal agent(s).

It should be capable of withstanding the rigors of mechanical shocks encountered in its production, packaging, shipping and dispensing.

An ideal tablet should be able to release the medicament(s) in body in predictable and reproducible manner.

\section{CONCLUSION}

Out of various drug delivery, buccal drug delivery is more permeable compare to others drug delivery. Even though the rectal, vaginal, and ocular mucosa all offer certain advantages, the poor patient acceptability associated with these sites renders them reserved for local applications rather than systemic drug administration. The buccal cavity, on the other hand, is highly acceptable by patients, the mucosa is relatively permeable with a rich blood supply, it is robust and shows short recovery times after stress or damage, and the virtual lack of Langerhans cells makes the buccal mucosa tolerant to potential allergens. Furthermore, buccal transmucosal drug delivery bypasses first pass effect and avoids pre-systemic elimination in the GI tract. These 
factors make the buccal mucosal cavity a very attractive and feasible site for systemic drug delivery.

\section{REFERENCES}

1. Karsten N., Olav H., Krzysztof I., Martin S., Ferry S., Johannes Z., Tobias L., "Occurrence and fate of the angiotensin-II receptor antagonist transformation product valsartan acid in the water cycle -A comparative study with selected $\beta$-blockers and the persistent anthropogenic waste water indicators carbamazepine and acesulfame", Elsevier water research, issue (47), 2013, 6650-6659.

2. Richa Sood, MS Rathore, Anil Sharma, Richa Thakur, Jayesh Chaudhari, Vijay Soni, "Immediate Release Antihypertensive Valsartan oral tablet", Journal of Scientific Research in Pharmacy, Review Article, 2012, 20-22.

3. Gandhi R.B. and Robinson J.R., "Oral cavity as a site for bioadhesive drug delivery", Advaned Drug Delivery, Revised, 13, 1994, 43-74.

4. Squier C.A. and Hall B.K., "The permeability of mammalian non-keratinized oral epithelia to horse raddish peroxidase applied in-vivo and in-vitro", Article on Oral Biology, 29, 1984, 45-50.

5. Hill M.W. and Squier C.A., "The permeability of oral palatal mucosa maintained in organ culture", Journal of Anatomy, 128, 1979, 169-178.

6. Tabak L.A., Levine M.J., Mandel I.D., and Ellison S.A., "Role of salivary mucins in the protection of the oral cavity", Journal of Oral Pathology, 11, 1982, 1-17.

7. Peppas N.A. and Buri P.A., "Surface, interfacial and molecular aspects of polymer bioadhesion on soft tissues", Journal of Controlled Release, 2, 1985, 257275.

8. Rathbone M., Drummond B., and Tucker I., "Oral cavity as a site for systemic drug delivery", Advanced Drug Delivery a Review, 13, 1994, 1-22.

9. Edgar W.M.,"Saliva: its secretion, composition and functions", Journal of Oral Pathology, 172, 1992, 305-312.

10. Bhardwaj N., Mukhopadhyay S., Tangri P. and Goswami L., "Buccal Mucosa: A Novelistic Route of Drug Delivery," International Journal Of Pharmaceutical And Chemical Sciences, Vol. 1 (3) Jul-Sep 2012, 837-849.

11. Ishida M., Nambu N., and Nagai T., "Mucosal dosage form of lidocaine for toothache using hydroxypropyl cellulose and carbopol", Chem. Pharma Bulletin, 30, 1982, 980-984.

12. Siegel I.A. and Gordon H.P., "Surfactant-induced increase of permeability of rat oral mucosa to nonelectolytes in-vivo", Arch. of Oral Biology, 30, 1985, 4347.
13. Shojaei A.H. and Lee X., "In-vitro permeation of acyclovir through porcine buccal mucosa", Proceedings of International Symposium on Controlled Release of Bioactive Materials, 23, 1996, 507-508.

14. Shojaei A.H. and Lee X., "Determination of transport route of acyclovir across buccal mucosa", Proceedings of International Symposium on Controlled Release of Bioactive Materials, 24, 1997, 427-428.

15. Manganaro A.M. and Wertz P.W., "The effects of permeabilizers on the in vitro penetration of propranolol through porcine buccal epithelium", Mil. Med., 161, 1996, 669-672.

16. Vanessa H., Vidya S., Katrusha H., Danica Vidovic J., Martin G., A. Ross K., Peter B. Lockhart, Lauren L. Patton, Stephen Porter, Martin H. Thornhill, "New developments and opportunities in oral mucosal drug delivery for local and systemic disease", Advanced Drug Delivery Reviews, 64, 2012, 16-28.

17. Gandhi R. and Robinson J., "Mechanisms of penetration enhancement for transbuccal delivery of salicylic acid", International Journal of Pharmaceutics, 85, 1992, 129-140.

18. Hoogstraate A.J., Verhoef J.C., Tuk B., Pijpe'rs A., Van leengoed, Vheijden J.H.M., Junjinger H.E., and Bodde H.E., "Buccal delivery of fluorescein isothiocyanatedextran 4400 and the peptide drug buserelin with glycodeoxycholate as an absorption enhancer in pigs", Journal of Controlled Release., 41, 1996, 77-84.

19. Wolany G.J.M., Munzer J., Rummelt A., and Merkle H.P., "Buccal absorption of Sandostatin (octreotide) in conscious beagle dogs", Proceedings of International Symposium on Controlled Release of Bioactive Materials, 17, 1990, 224-225.

20. Nakane S., Kakumoto M., Yulimatsu K., and Chien Y.W., “Oral mucosal delivery of LHRH:Pharmacokinetic studies of controlled and enhanced transmucosal permeation", Pharmaceytical Devices and Technologies, 1, 1996, 251-259.

21. Steward A., Bayley D.L., and Howes C., "The effect of enhancers on the buccal absorption of hybrid (BDBB) alpha-interferom", International Journal of Pharmacology, 104, 1994, 145-149.

22. Senel S., Hoogstraate A.J., Spies F., Verhoef J.C., Bosvan Geest A., Junginger H.E., and Bodde H.E., "Enhancement of in-vitro permeability of porcine buccal mucosa by bile salts: kinetic and histological studies", Journal of Controlled Release., 32, 1994, 4556.

23. Leipold H.R. and Quadros E., "Nicotine permeation through buccal mucosa", Proceedings of International Symposium on Controlled Release of Bioactive Materials, 20, 1993, 242-243. 
24. Beckett A.H. and Triggs E.J., "Buccal absorption of basic drugs and its application as an in-vivo model of passive drug transfer through lipid membranes", Journal of Pharmaceutics and Pharmacology, 19, 1967, 31-41.

25. Schurmann W. and Turner P., "A membrane model of the human oral mucosa as derived from buccal absorption performance and physicochemical properties of the beta-blocking drugs atenolol and propranolol", Journal of Pharmaceutics and Pharmacology, 30, 1978, 137-147.

26. Tucker I.G., "A method to study the kinetics of oral mucosal drug absorption from solutions", Journal of Pharmaceutics and Pharmacology, 40, 1988, 679-683.

27. Collins P., Laffoon J., and Squier C.A., "Comparative study of porcine oral epithelium", Journal of Dental Research, 60, 1981, 543-566.

28. Leung S.S. and Robinson J.R., "Polymer structure features contributing to mucoadhesion: II", Journal of Controlled Release, 12, 1990, 187-194.

29. Sanzgiri Y.D., Topp E.M., Benedetti L., and Stella V.J., "Evaluation of mucoadhesive properties of hyaluronic caid benzyl esters", International Journal of Pharmacology, 107, 1994, 91-97.

30. Lehr C.M., Bouwstra J.A., Schact E.H., and Junginger H.E., "In-vitro evaluation of mucoadhesive properties of chitosan and some other natural polymers", International Journal of Pharmacology, 78, 1992, 4348.
31. Park K. and Robinson J.R., "Bioadhesive polymers as platforms for oral-controlled drug delivery: method to study bioadhesion", International Journal of Pharmaceutics, 19, 1984, 107-127.

32. Nagai T. and Machida Y., "Buccal delivery systems using hydrogels", Advanced Drug Delivery Systems, 11, 1993, 179-191.

33. Satoh K., Takayama K., Machida Y., Suzuki Y., Nakagaki M., and Nagai T., "Factors affecting the bioadhesive property of tablets consisting of hydroxypropyl cellulose and carboxyvinyl polymer", Chemistry and Pharma Bulletin, 37, 1989, 1366-1368.

34. Gupta A., Garg S., and Khar R.K., "Interpolymer complexation and its effect on bioadhesion strength and dissolution characteristics of buccal drug delivery systems", International Journal of Pharmaceutics, 20, 1994, 315-325.

35. Anlar S., Capan, Y., Guven O., Gogus A., Dlakara T., and Hincal A.A., "Formulation and in-vitro/in-vivo evaluation of buccoadhesive morphine sulfate tablets", Pharmaceutical Researches, 11, 1994, 231-236

36. G. Sandeep and A. Navya, "Formulation and Development of Sustained Release Tablets of Diclofenac Sodium, International Journal of Advances In Pharmacy, Biology And Chemistry, Vol. 1(3), Jul- Sep, 2012, 300-304.

Source of Support: None declared.

Conflict of Interest: None declared.

For any question relates to this article, please reach us at: editor@globalresearchonline.net

New manuscripts for publication can be submitted at: submit@globalresearchonline.net and submit_ijpsrr@rediffmail.com 